\title{
Simulations of Massive Star Cluster Formation and Feedback in Turbulent Giant Molecular Clouds
}

\author{
Elizabeth Harper-Clark ${ }^{1,2}$ and Norman Murray ${ }^{1}$ \\ ${ }^{1}$ Canadian Institute for Theoretical Astrophysics, Toronto, Ontario, Canada \\ 2 email: h-clark@cita.utoronto.ca
}

\begin{abstract}
Using the AMR code ENZO we are simulating the formation of massive star clusters within turbulent Giant Molecular Clouds (GMCs). Here we discuss the simulations from the first stages of building realistic turbulent GMCs, to accurate star formation, and ultimately comprehensive feedback. These simulations aim to build a better understanding of how stars affect GMCs, helping to answer the questions of how long GMCs live and why only a small fraction of the GMC gas becomes stars.
\end{abstract}

Keywords. stars: formation, galaxies: ISM, galaxies: star clusters, ISM: clouds, ISM: bubbles, ISM: structure, ISM: jets and outflows, turbulence, radiative transfer, and MHD

\section{Introduction}

Within the Milky Way there are $~ 300$ Giant Molecular Clouds (GMCs) with masses of the order a million solar masses. For these GMCs, the average star formation rate per free-fall time $\left(\mathrm{SFR}_{f f}\right)$ is $2 \%$ (Kennicutt 1998). However, the majority of the star formation is contained within fewer than 50 of these GMCs (Murray \& Rahman 2010 and Murray 2010). The small fraction of GMCs with high $\mathrm{SFR}_{f f}$ suggests that the $\mathrm{SFR}_{f f}$ of a GMC changes over its lifetime. We propose that the star formation rate increases over a cloud's lifetime, as shown in cartoon form in Fig. 1. The GMCs with highest

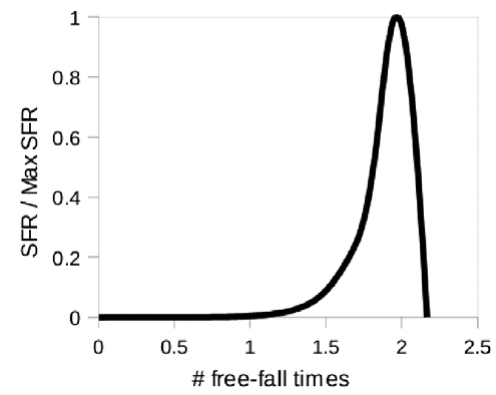

Figure 1. A cartoon of our propose star formation rate per free-fall time over an individual clouds lifetime ending with its destruction at $\sim 2$ free fall times.

$\mathrm{SFR}_{f f}$ are seen to be in the process of disruption, Fig. 2. Once a large star cluster forms it will blow apart its parent GMC (Harper-Clark and Murray 2009 and Fig. 2), thus the last period of star formation is likely the GMCs most rapid. 


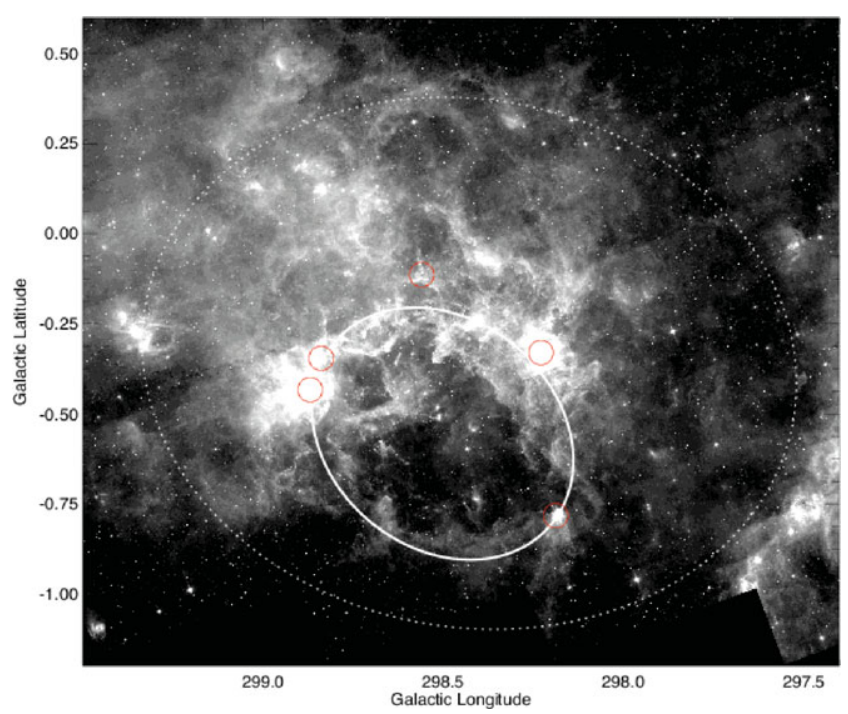

Figure 2. A Spitzer Glimpse image of G298-0.34, a GMC in the process of being blow apart by an invisible central massive star cluster (Murray \& Rahman 2010). The small red dashed ellipses show compact HII regions from triggered star formation. The solid oval is the bubble wall and the large dotted oval is the extent of the WMAP source, suggesting the hot gas is leaking from the bubble (Harper-Clark and Murray 2009).

\section{Code}

The high-resolution Eulerian AMR code ENZO (Bryan \& Norman 1997 and O'Shea et al. 2004 etc.) contains all necessary physics for accurate testing of turbulent GMC gravitational collapse. We are using the developer's version which includes MHD, protostellar jets, radiative transfer, and an alternative hydro solver using a total variation diminishing second order Runge-Kutter scheme for time-integration (see Wang and Abel 2009 and Wise and Abel 2008 for details).

\section{Simulation Set-up}

To model an entire GMC we set up a cubic simulation box $128 \mathrm{pc}$ wide. The top grid has dimensions $256^{3}(0.5 \mathrm{pc})$ and is refined by 2 for up to 8 levels (effective resolution of $65536,0.002 \mathrm{pc}, 400 \mathrm{AU}$ ). Refinement is based upon Jeans' length or the presence of stars. Subgrids are set up so that each star is always surrounded by at least $0.03 \mathrm{pc}$ of maximally refined region in all directions.

The initial velocities of the gas are set by a seeded random distribution fitting a Larson turbulent spectrum with $2 \leqslant k \leqslant 32$ (e.g., Mac Low et al. 1998) at Mach 9 and decay appropriately.

The initial density distribution is a cored isothermal sphere with radius of $44.8 \mathrm{pc}$. The size of the core can be varied to get different distributions whilst maintaining a mass of a million solar masses of gas within the cloud. Although a smooth spherically symmetric density distribution is not realistic for a GMC the random velocities cause filamentary structures to form rapidly and before any stars form the densities look much more realistic (Fig. 3).

Stars are formed according to the Truelove criterion (Truelove et al. 1997). Once the Jeans' length $\left(l_{J}\right)$ is less than 4 maximum refinement level cell widths only the mass 


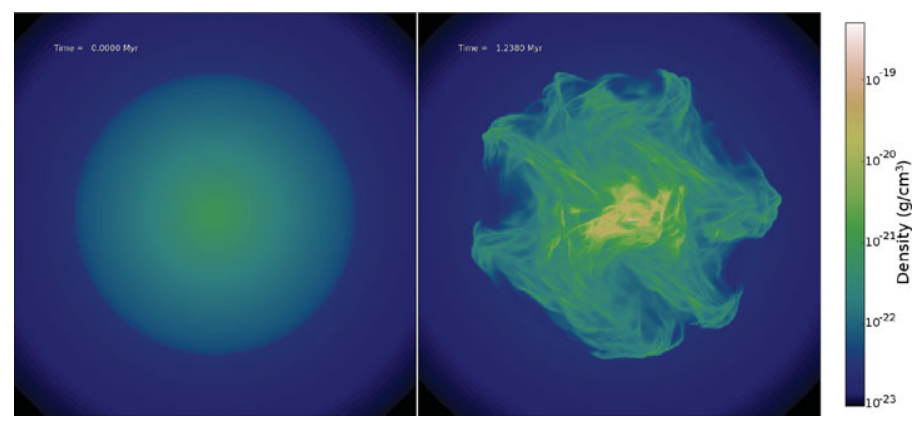

Figure 3. Initial density distributions. Left: time $=0$ the cloud is a spherically symmetric cored isothermal sphere. Right: after 1.238 million years the turbulence has formed a filamentary structure and the first stars are about to form.

needed to bring the Jeans length back to 4 cell widths is taken out of the cell and forms a sink particle conserving momentum and mass.

\section{Preliminary Results}

While the simulations have only run as far as forming the first star cluster to date, there are some interesting results from our simulations worth discussing.

\subsection{Local conditions for star formation}

By construction the stars in our simulation form where the Jeans length becomes less than 0.008 pc. Upon close inspection this typically occurs where three or more filaments of inflow meet or when two filaments collide, as shown in Fig. 4.
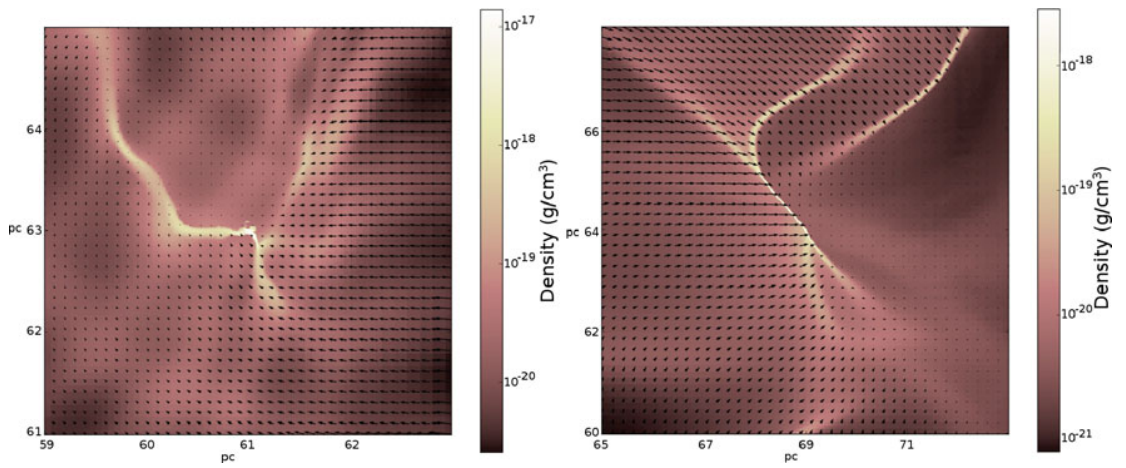

Figure 4. Density slices of a core about to form a star from hydrodynamic simulations showing the velocities of the surrounding gas (black arrows). Left: three or more filaments of inflow meeting. Right: just after two filaments have collided

\subsection{Location of first stars within the GMC}

Precisely where within a GMC the first stars form depends strongly upon the central concentration of the density. For example Fig. 5 shows two different GMCs with identical initial velocity distribution, the same mass but different radial density profiles. Both clouds are cored isothermal spheres, one with density varying as: $\rho(r)=4.25 \rho_{c} /(1+$ $\left.\left(9 r / r_{c l}\right)^{2}\right)$ so half mass radius $=0.57$ (left in Fig. 5$)$ and the other: $\rho(r)=1.05 \rho_{c} /(1+$ $\left.\left(4 r / r_{c l}\right)^{2}\right)$ so half mass radius $=0.63$ (right in Fig. 5) where $\rho_{c}$ is a set density and $r_{c l}$ is the radius of the cloud. 


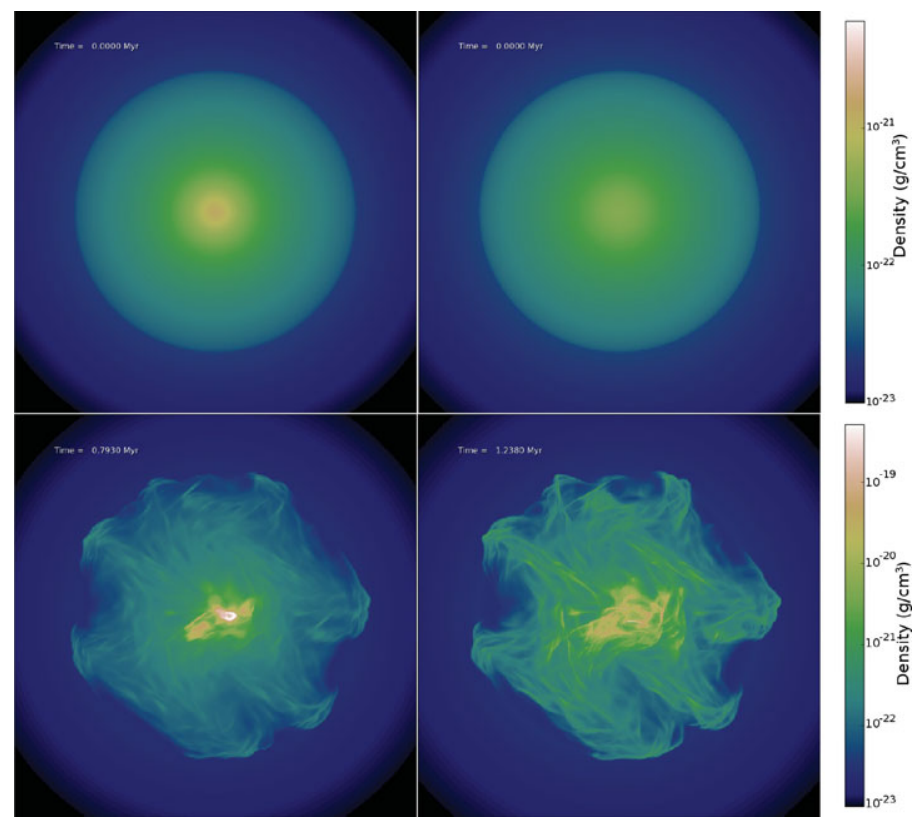

Figure 5. Two clouds with different central concentrations but identical initial velocity distribution and total mass. The top two images show the initial density when spherically symmetric and the bottom images are the corresponding clouds just before the first stars form in each. Please note the different colourbars between the top and bottom images (colourbars on right).

\section{Future Work}

These results provide an enticing glimpse into a field where much work is still needed. It is important to investigate further where stars first form with different turbulent seeds and different density profiles to understand what effect the initial conditions have upon results. Also, how does star formation rate vary with time within simulations with no feedback and with some or all feedbacks (jets, HII, radiation pressure, winds, supernovae). Additionally, How do magnetic fields affect the formation of stars and subsequent evolution of the clouds?

\section{References}

Bryan, G. L. and Norman, M. L. Mar. 1997, In "Workshop on Structured Adaptive Mesh Refinement Grid Methods", ed. N. Chrisochoides

Harper-Clark, E. \& Murray, N. 2009, ApJ, 693, 1696

Kennicutt, R. C. $1998, A p J, 498,541$

Mac Low, M.-M., Klessen, R.S., Burkert, A., \& Smith, M.D. 1998, Phys. Rev. Lett., 80, 2754

Murray, N. 2010, submitted

Murray, N. \& Rahman, M. 2010, ApJ, 709, 424

O'Shear, B.W. et al. 2004, In "Adaptive Mesh Refinement - Theory and Applications," Eds. T. Plewa, T. Linde \& V. G. Weirs, Springer Lecture Notes in Computational Science and Engineering.

Truelove, J. K. et al 2009, ApJL, 489, 179

Wang, P. \& Abel, T. 2010, ApJ, 696, 96

Wise, J. \& Abel, T. 2008, ApJ, 685, 40 